\section{Roles of GRK2 in Cell Signaling Beyond GPCR Desensitization: GRK2-HDAC6 Interaction Modulates Cell Spreading and Motility}

\author{
Petronila Penela, ${ }^{1,2 \star}$ Vanesa Lafarga,,${ }^{1,2}$ Olga Tapia, ${ }^{3}$ Verónica Rivas, ${ }^{1,2}$ \\ Laura Nogués, ${ }^{1,2}$ Elisa Lucas, ${ }^{1,2}$ Rocío Vila-Bedmar, ${ }^{1,2}$ Cristina Murga, ${ }^{1,2}$ \\ Federico Mayor Jr., ${ }^{1,2 *} \dagger$
}

G protein-coupled receptor kinase 2 (GRK2) is a ubiquitous, essential protein kinase that is emerging as an integrative node in many signaling networks. Moreover, changes in GRK2 abundance and activity have been identified in several inflammatory, cardiovascular disease, and tumor contexts, suggesting that those alterations may contribute to the initiation or development of pathologies. GRKs were initially identified as key players in the desensitization and internalization of multiple G protein-coupled receptors (GPCRs), but GRK2 also phosphorylates several non-GPCR substrates and dynamically associates with a variety of proteins related to signal transduction. Ongoing research in our laboratory is aimed at understanding how specific GRK2 interactomes are orchestrated in a stimulus-, context-, or cell typespecific manner. We have recently identified an interaction between GRK2 and histone deacetylase 6 (HDAC6) that modulates cell spreading and motility. HDAC6 is a major cytoplasmic $\alpha$-tubulin deacetylase that is involved in cell motility and adhesion. GRK2 dynamically and directly associates with and phosphorylates HDAC6 to stimulate its $\alpha$-tubulin deacetylase activity at specific cellular localizations such as the leading edge of migrating cells, thus promoting local tubulin deacetylation and enhanced motility. GRK2-HDAC6-mediated regulation of tubulin acetylation also modulates cellular spreading. This GRK2-HDAC6 functional interaction may have important implications in pathological contexts related to epithelial cell migration.

\section{Presentation Notes}

Slide 1: Science Signaling logo

The slideshow and notes for this presentation are provided by Science Signaling (www.sciencesignaling.org).

Slide 2: Roles of GRK2 in cell signaling beyond GPCR desensitization: GRK2HDAC6 interaction modulates cell spreading and motility

This talk briefly reviews cellular functions of $\mathrm{G}$ protein-coupled receptor 2 (GRK2) beyond its canonical role in $\mathrm{G}$ protein-coupled receptor (GPCR) desensitization and focuses on the description of a recently identified GRK2-HDAC6 interaction that modulates epithelial cell spreading and motility.

'Departamento de Biología Molecular and Centro de Biología Molecular "Severo Ochoa", Universidad Autónoma de Madrid, 28049 Madrid, Spain. 'Instituto de Investigación Sanitaria La Princesa, 28006 Madrid, Spain. ${ }^{3}$ Department of Anatomy and Cell Biology, University of Cantabria-IFIMAV, Santander, Spain.

${ }^{*}$ Corresponding authors. E-mail: fmayor@cbm. uam.es (F.M.); ppenela @ cbm.uam.es (P.P.)

†Presenter. E-mail: fmayor@cbm.uam.es lator Ral-GDS, the ubiquitin ligase $\mathrm{Mdm} 2$, and components of the nuclear factor $-\kappa \mathrm{B}$
$(\mathrm{NF}-\kappa \mathrm{B})$ and mitogen-activated protein kinase (MAPK) signaling pathways $(1,3)$. The signalosomes orchestrated by arrestins would allow signal propagation at defined locations, contributing to the overall cellular response to the presence of an agonist.

It should be stressed, however, that the cellular role of GRKs is not limited to promoting $\beta$-arrestin binding to activated GPCRs. GRKs are multidomain proteins with diverse cellular functions, the expression and function of which are tightly regulated and altered in several cardiovascular, inflammatory, and tumor pathologies, thus suggesting that these changes may contribute to the onset or development of disease (4).

Slide 5: GRK2 is a key node in signal transduction pathways: The complex GRK2 interactome

GRK2, an ubiquitous and essential member of the GRK family, is emerging as a key, integrative node in many signaling networks because it has an extensive repertoire of functional interactions (its interactome). Besides phosphorylating activated GPCRs, GRK2 is functionally related to several members of the receptor tyrosine kinase family, phosphorylates a variety of nonreceptor substrates, and dynamically interacts with other important signal transduction partners in a manner that is independent of its kinase activity $(4,5)$. For instance, as emphasized in this slide, in the past few years our group has reported GRK2 interactions with the mitogen-activated or extracellular signal-regulated protein kinase kinase MEK (6), the MAPK p38 (7), the ARF (adenosine diphosphate ribosylation factor) GTPase (guanosine triphosphatase)activating protein GIT1 (8), Mdm2 (9), the prolyl-isomerase Pin1 (10), and insulin receptor substrate 1 (IRS1) (11). Some of these interactions underlie the participation of GRK2 in basic cellular processes such as cell cycle progression (10), cell migration (8), and insulin resistance (11).

Slide 6: Questions arising from the complexity of the GRK2 interactome In the face of such a complex interactome, research in this field is aimed at addressing the following questions: How are the different networks of potential GRK2 functional interactions orchestrated in a stimulus-, context-, or cell type-specific manner? Which is the relevant GRK2 interactome in a given cell type and physiological situation? What are the physiological and pathological implications of altered GRK2 abundance or activity? 
Slide 7: Examples of relevant GRK2 interactomes reported in given cell types and physiological settings

Recent research by different laboratories ( 4 , 12) has started to define the distinct relevant functional interactions of GRK2 - both related and unrelated to its canonical role as GPCR modulator - underlying its participation in physiological or pathological processes in different cell types. For example, in lymphocytes GRK2 interacts with chemokine receptors and modulates the interaction between MEK and ERK (extracellular signalregulated kinase) and the status of p38 activation, so the decreased GRK2 abundance reported in inflammatory contexts would promote enhanced signaling through these pathways. On the other hand, in adipocytes GRK2 can act as an inhibitor of insulin-mediated signaling by interacting with $\mathrm{G}_{\mathrm{q}}$ and $\mathrm{G}_{11}$ (13) or by forming dynamic complexes with IRS1, which is consistent with a role for GRK2 in insulin resistance and obesity (11).

Slide 8: GRK2 interactome in epithelial cell migration

Our group is actively investigating the role of GRK2 in epithelial cell migration and has recently unveiled a previously undocumented GRK2-HDAC6 interaction that modulates cell spreading and motility (14). In epithelial cells, locomotion is initiated by chemoattractants acting through either GPCR or tyrosine kinase receptors that trigger downstream signals leading to cell polarization, projection of cytoplasmic extensions (pseudopodia or lamellipodia) that are enriched in actin and devoid of organelles at the leading edge, and cell body translocation forward by modulation of the actin cytoskeleton and microtubule (MT) dynamics (15). Acetylation and deacetylation of the MT component $\alpha$-tubulin has been suggested to play a prominent role in cell migration and adhesion (16).

Slide 9: HDAC6 is a cytoplasmic deacetylase

HDAC6 is a class IIa cytoplasmic histone deacetylase that can deacetylate substrates such as $\alpha$-tubulin, cortactin, and Hsp90 to modulate diverse cellular processes such as agressome or stress granule (SG) formation, cell spreading, and migration. In particular, HDAC6 up-regulation and concomitant decreased tubulin acetylation enhance the motility of different cell types including fibroblasts (17) and breast cancer cells $(18,19)$.

Slide 10: GRK2 positively regulates epithelial cell migration

We have recently shown that GRK2 positively regulates integrin-dependent motility in epithelial cells and fibroblasts (8). This effect was due in part to GRK2's kinase activity-independent modulation of the scaffold function of GIT-1 in the context of Rac-PAK-MEK-ERK1/2 pathway activation, thus promoting $\mathrm{F}$-actin remodeling at the cell periphery and focal adhesion turnover. However, we also noted that increased GRK2 abundance enhanced cell migration on fibronectin (FN) in a way that required the kinase activity of GRK2. Whereas increased abundance of wild-type (WT) GRK2 enhanced migration of HeLa cells in a wound-healing assay, wound closure was blocked by expression of a catalytically inactive point mutant $\left(\mathrm{GRK} 2^{\mathrm{K} 220 \mathrm{R}}\right)$ or upon GRK2 deletion in mouse embryonic fibroblasts (MEFs) by using a Cre-Lox system (left).

Slide 11: GRK2 abundance modulates the extent of tubulin acetylation in a kinase-activity dependent manner

Are GRK2 and HDAC6 functionally related in this positive modulation of epithelial cell migration? Interestingly, GRK2 down-regulation in MEFs derived from hemizygous $G R K 2^{+/-}$mice clearly showed enhanced tubulin acetylation as compared with that of WT MEFs, without apparent changes in the expression of either HDAC6 or SIRT2 deacetylases (top left). Similarly, deletion of GRK2 in MEFs by using a Cre-Lox system (bottom left) or GRK2 down-regulation triggered by means of RNA interference in HeLa cells (middle) led to increased accumulation of acetylated tubulin in parallel with reduced motility. Notably, acetylated tubulin markedly accumulated in HeLa cells that stably overexpressed a transgene encoding either a catalytically inactive GRK2 (K1 cells) or a version of GRK2 bearing a mutation at the $\mathrm{S} 670$ regulatory site (GRK2 $2^{\mathrm{S} 670 \mathrm{~A}}$; A1 cells) and correlated with the impaired ability of these cells to migrate toward both mechanical (wounding) and chemotactic cues (top right).

Slide 12: The effect of GRK2 on cell migration is counteracted by tubacin, a specific inhibitor of HDAC6-dependent deacetylation of tubulin.

In support of GRK2-mediated enhanced cell migration requiring $\alpha$-tubulin acetylation-deacetylation cycling, the presence of the general HDAC inhibitor TSA or of tubacin - a specific inhibitor of HDAC6 tubulin (but not cortactin) deacetylase activity - counteracted the ability of increased GRK2 abundance to stimulate cell motility. These data suggested that GRK2 specifi- cally facilitates HDAC6 activity toward tubulin in order to promote deacetylation and enhanced cell motility and posed the question of whether this was a result of a direct or indirect interaction between GRK2 and HDAC6.

Slide 13: HDAC6 and GRK2 colocalize in the leading edge of migrating cells, coincident with decreased local tubulin acetylation

Both GRK2 and HDAC6 are recruited to chemoattractant-induced pseudopodia, and the proteins colocalized in the leading edge of polarized HeLa cells that were directionally migrating to close a scratch wound. Interestingly, acetylated MTs displayed an asymmetric distribution, with an increased density on the side of the cell closest to the wound, but were excluded from the lamellipodium at the leading edge. Such non-overlapping distribution of HDAC6 and acetylated MTs at the cell border is consistent with a role for HDAC6mediated deacetylation in motility and with active tubulin deacetylation taking place at the leading edge.

Slide 14: HDAC6 and GRK2 coimmunoprecipitate and are able to interact directly A functional interaction between GRK2 and HDAC6 is further backed by the following observations: First, GRK2 coimmunoprecipitates with hemagglutinin (HA)-tagged HDAC6 in cells transiently transfected with constructs encoding these proteins (A). Secondly, coimmunoprecipitation of endogenous HDAC6 and GRK2 can also be detected in cytoplasmatic extracts from HeLa cells (B), indicating a specific association of these proteins under steady-state physiological conditions. Last, the GRK2-HDAC6 association does not require other protein intermediates, as indicated by the direct binding of recombinant GRK2 and glutathione $S$-transferase (GST)-tagged HDAC6 (C). Overall, our data support the notion that GRK2 directly interacts with HDAC6 at the cell periphery to positively regulate its activity to promote local tubulin deacetylation, which would contribute to a gradient of MT instability that seems to be critical for migration $(20,21)$. The next question was how GRK2 stimulated HDAC6 activity.

Slide 15: GRK2 phosphorylates HDAC6 to stimulate tubulin deactetylase activity In vitro kinase assays revealed that purified GST-HDAC6 was readily phosphorylated by recombinant GRK2 with high affinity (left). Notably, in vitro deacetylation assays showed that preincubation with GRK2 under phosphorylation-permissive conditions 
clearly enhanced both the extent and kinetics of HDAC6-mediated $\alpha$-tubulin deacetylation (right), indicating that GRK2-mediated phosphorylation was the mechanism underlying its direct positive modulatory effect on HDAC6 activity.

Slide 16: HDAC6 mutants with impaired phosphorylation by GRK2 fail to mimic the enhanced chemotactic motility promoted by WT HDAC6 in HeLa cells

We set out to identify the site (or sites) of HDAC6 phosphorylation by GRK2. In vitro and cellular assays (14) revealed serines 1060, 1062, and 1069 of HDAC6 as important phosphoacceptor sites and indicated that phosphorylation of HDAC6 at these residues was necessary for full tubulin deacetylase activity. Moreover, mutant HDAC6 proteins in which either of two of these serine residues had been mutated to alanines (S1060A and S1062A) showed impaired phosphorylation by GRK2, and as shown in this slide, HeLa cells expressing constructs encoding these mutant HDAC6s failed to mimic the enhanced chemotactic motility promoted by WT HDAC6, which is similar to the effect of a tubulin-deacetylase inactive mutant (HDAC6-DD). These data strongly suggest that HDAC6 phosphorylation by GRK2 plays an important role in the positive modulation of cell motility by these proteins.

Slide 17: GRK2-dependent regulation of HDAC6 activity modulates cell spreading kinetics

We have also investigated the potential involvement of GRK2-mediated HDAC6 modulation in cell spreading, which is also affected by changes in MT dynamics and acetylation $(22,23)$. To summarize our published data (14), we found that downregulation of GRK2 or expression of either $G R K 2^{2^{\mathrm{S} 670 \mathrm{~A}}}$ or $G R K 2^{\mathrm{K} 220 \mathrm{R}}$ notably altered the normal spreading pattern of HeLa cells on FN-coated surfaces. These cells displayed an enhanced initial spreading, with earlier expansion of the MT network and a higher proportion of acetylated MTs as compared with WT cells, suggesting that modulation of HDAC6-mediated MT acetylation by GRK2 regulates the adhesion-independent early phase of cell spreading. However, unlike cells lacking tubulin deacetylase activity, $G R K 2^{\mathrm{S} 670 \mathrm{~A}}$ - or $G R K 2^{\mathrm{K} 220 \mathrm{R}}$-expressing cells did not attain a larger final cellular area than that of WT cells, suggesting the involvement of GRK2 in additional, non-HDAC6-related processes during the late adhesion-dependent phase of spreading (14).
Interestingly, many MTs were found in membrane protrusions at the cell periphery during the cell spreading, which is similar to the dynamic pioneer MTs observed at the lamellipodium of motile cells. These MTs were less acetylated at their distal than at their proximal ends (magnified images) during both early and late spreading of parental HeLa and cells overexpressing WT GRK2 (wt5 cells), but only during early spreading in HeLa-A1 and HeLa-K1 cells. Therefore, active tubulin deacetylation seems to be taking place at the cell edge late in spreading, in a process that requires GRK2 to be catalytically competent toward HDAC6 for the cell to spread fully. In summary, GRK2-HDAC6-mediated control of tubulin acetylation plays different modulatory roles during distinct phases of the cell spreading process.

Slide 18: Regulation of HDAC6 activity by GRK2 is dependent on GRK2 phosphorylation status at Ser ${ }^{670}$

It is important to stress that the modulatory effect of GRK2 on HDAC6 can be dynamically regulated by the phosphorylation status of GRK2 at serine 670 (S670). As described in previous slides, tubulin acetylation was augmented in cells producing either the catalytically inactive GRK2 ${ }^{\mathrm{K} 220 \mathrm{R}}$ protein or GRK2 ${ }^{\mathrm{S} 670 \mathrm{~A}}$, a mutant that retains catalytic activity toward GPCRs and tubulin. The inability of these mutants to modulate HDAC6 was not due to defective binding to HDAC6 (14). Remarkably, we observed that GRK2 $2^{\mathrm{S} 670 \mathrm{~A}}$ showed a noticeably reduced ability to phosphorylate HDAC6 as compared with WT GRK2 but did not show any defects in phosphorylating other GRK2 canonical substrates (top left). Interestingly, phosphorylation of GRK2 at S670 rapidly increases in response to promigratory stimuli coincident with tubulin deacetylation (top right) and specifically increases where it colocalizes with HDAC6 in pseudopodia of motile cells (bottom confocal images). These observations suggest that phosphorylation of GRK2 at S670 in the cell leading edge would facilitate localized phosphorylation of HDAC6 in situ.

Slide 19: GRK2 phosphorylation at the S670 regulatory site acts as a switch that specifically modulates its ability to phosphorylate HDAC6

Overall, our results indicate that phosphorylation of GRK2 at the S670 regulatory site acts as a key switch that specifically modulates its ability to phosphorylate HDAC6, thus affecting HDAC6 activity. The inabil- ity of GRK2 $2^{\mathrm{S} 670 \mathrm{~A}}$ to phosphorylate HDAC6, but not other substrates, constitutes the first example that phosphorylation at $\mathrm{S} 670$ could switch GRK2's substrate repertoire. We propose that dynamic GRK2 phosphorylation at the leading edge induced by different pro-migratory stimuli would translate into dynamic, local HDAC6-mediated deacetylation of tubulin at the plus-ends of MTs, thus helping to maintain the cortical polarization that underlies pseudopodia extension and directed migration.

As depicted in the lower schematic model, we postulate that upon chemotactic receptor activation, GRK2 would be recruited in a G $\beta \gamma$-dependent manner to the lamellipodium plasma membrane, where chemokine receptor stimulation would promote GRK2 phosphorylation at $\mathrm{S} 670$ by MAPK. This in turn would switch on the ability of GRK2 to phosphorylate colocalized HDAC6, resulting in greater local deacetylase activity toward tubulin. The presence of highly dynamic, hypoacetylated MTs at the lamellipodium would stimulate cortical F-actin polymerization and promote cell motility (14).

Slide 20: Dynamic modulation of GRK2 networks by phosphorylation

Together with other recent results in our laboratory, our data further stress the notion that GRK2 phosphorylation by other kinases could act as signaling switches to govern GRK2's substrate specificity and its interactions with cellular partners. For instance, phosphorylation of GRK2 at specific tyrosine residues by c-Src enhances its interaction with the scaffold protein GIT1, whereas phosphorylation by MAPK at S670 shows the opposite effect $(8)$. In the context of the cell cycle, Cdk2-mediated GRK2 phosphorylation at $\mathrm{S} 670$ promotes its interaction with the prolyl-isomerase Pin1, which is instrumental for the transient down-regulation of GRK2 abundance required for normal cell cycle progression (10). In this way, the phosphorylation status of GRK2 would be a key factor underlying the dynamic and stimuli-specific modulation of GRK2 networks.

Slide 21: Relevant GRK2 interactome in epithelial cell migration and physiopathological implications

In conclusion, we have identified a functional interaction of GRK2 with HDAC6 that plays an important role in cell spreading and motility, thus adding another component to the GRK2 interactome relevant to epithelial cell migration. We hypothesize that this GRK2-HDAC6 relationship may have important implications in the 
context of cancer cell migration and invasion. HDAC6 activity and reduced tubulin acetylation have been associated with malignant transformation and invasive motility in breast cancer $(18,24)$. GRK2 abundance is up-regulated in several malignant mammary cell lines compared with normal cells $(12,25)$, and increased GRK2 abundance enhances epithelial cell motility upon integrin and GPCR engagement (8) and leads to reduced DNA damage-induced $\mathrm{p} 53$ responsiveness (10). Therefore, it is tempting to suggest that concurrent HDAC6 and GRK2 up-regulation in human tumor malignancies would favor aberrant cell motility, adhesion, and transformation.

Slide 22: Acknowledgments

Most of the work regarding the functional interaction between HDAC6 and GRK2 in epithelial cell migration was performed by postdoctoral fellow Vanesa Lafarga in our laboratory, with the leadership of my senior collaborator Petronila Penela, and involved collaboration with Olga Tapia of the Department of Anatomy and Cell Biology, University of Cantabria-Spain. Other coauthors from my group have participated in additional lines of research that have unveiled more roles for GRK2 in cell signaling in addition to its canonical role in GPCR desensitization. Our laboratory is funded by grants from Ministerio de Educación y Ciencia (SAF2011-23800), Fundación Ramón Areces, Instituto de Salud Carlos III (RD06-0014/0037 and PI11/00859), and Comunidad de Madrid (S2011/BMD2332).

Editor's Note: This contribution is not intended to be equivalent to an original research paper. Note, in particular, that the text and associated slides have not been peer-reviewed.

\section{References}

1. R. T. Premont, R. R. Gainetdinov, Physiological roles of $\mathrm{G}$ protein-coupled receptor kinases and arrestins. Annu. Rev. Physiol. 69, 511-534 (2007).

2. C. A. Moore, S. K. Milano, J. L. Benovic, Regulation of receptor trafficking by GRKs and arrestins.
Annu. Rev. Physiol. 69, 451-482 (2007)

3. S. K. Shenoy, R. J. Lefkowitz, $\beta$-Arrestin-mediated receptor trafficking and signal transduction. Trends Pharmacol. Sci. 32, 521-533 (2011).

4. P. Penela, C. Murga, C. Ribas, V. Lafarga, F. Mayor, Jr., The complex $G$ protein-coupled receptor kinase 2 (GRK2) interactome unveils new physiopathological targets. Br. J. Pharmacol. 160, 821-832 (2010)

5. C. Ribas, P. Penela, C. Murga, A. Salcedo, C. García-Hoz, M. Jurado-Pueyo, I. Aymerich, F. Mayor, Jr., The G protein-coupled receptor kinase (GRK) interactome: role of GRKs in GPCR regulation and signaling. Biochim. Biophys. Acta 1768, 913922 (2007).

6. M. C. Jiménez-Sainz, C. Murga, A. Kavelaars, M. Jurado-Pueyo, B. F. Krakstad, C. J. Heijnen, F. Mayor, Jr, A. M. Aragay, G protein-coupled receptor kinase 2 negatively regulates chemokine signaling at a level downstream from $\mathrm{G}$ protein subunits. Mol. Biol. Cell 17, 25-31 (2006).

7. S. Peregrin, M. Jurado-Pueyo, P. M. Campos, V. Sanz-Moreno, A. Ruiz-Gomez, P. Crespo, F. Mayor, Jr, C. Murga, Phosphorylation of p38 by GRK2 at the docking groove unveils a novel mechanism for inactivating p38MAPK. Curr. Biol. 16, 20422047 (2006)

8. P. Penela, C. Ribas, I. Aymerich, N. Eijkelkamp, O. Barreiro, C. J. Heijnen, A. Kavelaars, F. SánchezMadrid, F. Mayor, Jr., G protein-coupled receptor kinase 2 positively regulates epithelial cell migration. EMBO J. 27, 1206-1218 (2008).

9. L. Nogués, A. Salcedo, F. Mayor, Jr, P. Penela, Multiple scaffolding functions of beta-arrestins in the degradation of $\mathrm{G}$ protein-coupled receptor kinase 2. J. Biol. Chem. 286, 1165-1173 (2011).

10. P. Penela, V. Rivas, A. Salcedo, F. Mayor, Jr., G protein-coupled receptor kinase 2 (GRK2) modulation and cell cycle progression. Proc. Natl. Acad. Sci. U.S.A. 107, 1118-1123 (2010).

11. L. Garcia-Guerra, I. Nieto-Vazquez, R. VilaBedmar, M. Jurado-Pueyo, G. Zalba, J. Díez, C. Murga, S. Fernández-Veledo, F. Mayor, Jr, M. Lorenzo, $G$ protein-coupled receptor kinase 2 plays a relevant role in insulin resistance and obesity. Diabetes 59, 2407-2417 (2010).

12. F. Mayor, P. Penela, C. Ribas, C. Murga, "The complex role of $\mathrm{G}$ protein-coupled receptor kinase 2 (GRK2) in cell signalling: Beyond GPCR desensitization," in "G protein-coupled receptors: From structure to function (Jesús Giraldo and Jean-Philippe Pin, Eds.). R. Soc. Chem. UK, RSC Drug Disc. Ser. 8, 316-334 (2011).

13. I. Usui, T. Imamura, H. Satoh, J. Huang, J. L. Babendure, C. J. Hupfeld, J. M. Olefsky, GRK2 is an endogenous protein inhibitor of the insulin signaling pathway for glucose transport stimulation. EMBO J. 23, 2821-2829 (2004).

14. V. Lafarga, I. Aymerich, O. Tapia, F. Mayor, Jr, P. Penela, A novel GRK2/HDAC6 interaction modulates cell spreading and motility. EMBO J. 31, 856-869 (2012).

15. A. Berzat, A. Hall, Cellular responses to extracellu- lar guidance cues. EMBO J. 29, 2734-2745 (2010).

16. C. Creppe, L. Malinouskaya, M. L. Volvert, M. Gillard, P. Close, O. Malaise, S. Laguesse, I. Cornez, S. Rahmouni, S. Ormenese, S. Belachew, B. Malgrange, J. P. Chapelle, U. Siebenlist, G. Moonen, A. Chariot, L. Nguyen, Elongator controls the migration and differentiation of cortical neurons through acetylation of alpha-tubulin. Cell 136, 551-564 (2009).

17. C. Hubbert, A. Guardiola, R. Shao, Y. Kawaguchi, A. Ito, A. Nixon, M. Yoshida, X. F. Wang, T. P. Yao, HDAC6 is a microtubule-associated deacetylase. Nature 417, 455-458 (2002).

18. K. Azuma, T. Urano, K. Horie-Inoue, S. Hayashi, R. Sakai, Y. Ouchi, S. Inoue, Association of estrogen receptor alpha and histone deacetylase 6 causes rapid deacetylation of tubulin in breast cancer cells. Cancer Res. 69, 2935-2940 (2009).

19. S. Saji, M. Kawakami, S. Hayashi, N. Yoshida M. Hirose, S. Horiguchi, A. Itoh, N. Funata, S. L. Schreiber, M. Yoshida, M. Toi, Significance of HDAC6 regulation via estrogen signaling for cell motility and prognosis in estrogen receptor-positive breast cancer. Oncogene 24, 4531-4539 (2005).

20. Y. Zilberman, C. Ballestrem, L. Carramusa, R. Mazitschek, S. Khochbin, A. Bershadsky, Regulation of microtubule dynamics by inhibition of the tubulin deacetylase HDAC6. J. Cell Sci. 122 3531-3541 (2009).

21. S. E. Siegrist, C. Q. Doe, Microtubule-induced cortical cell polarity. Genes Dev. 21, 483-496 (2007).

22. S. Rhee, H. Jiang, C. H. Ho, F. Grinnell, Microtubule function in fibroblast spreading is modulated according to the tension state of cell-matrix interactions. Proc. Natl. Acad. Sci. U.S.A. 104, 5425-5430 (2007)

23. A. D. Tran, T. P. Marmo, A. A. Salam, S. Che, E. Finkelstein, R. Kabarriti, H. S. Xenias, R. Mazitschek, C. Hubbert, Y. Kawaguchi, M. P. Sheetz, T. P. Yao, J. C. Bulinski, HDAC6 deacetylation of tubulin modulates dynamics of cellular adhesions. J. Cell Sci. 120, 1469-1479 (2007).

24. J. Suzuki, Y. Y. Chen, G. K. Scott, S. Devries, K. Chin, C. C. Benz, F. M. Waldman, E. S. Hwang, Protein acetylation and histone deacetylase expression associated with malignant breast cancer progression. Clin. Cancer Res. 15, 3163-3171 (2009).

25. A. Salcedo, F. Mayor, Jr, P. Penela, Mdm2 is involved in the ubiquitination and degradation of $\mathrm{G}$ protein-coupled receptor kinase 2. EMBO J. 25 4752-4762 (2006).

10.1126/scisignal.2003098

Citation: P. Penela, V. Lafarga, O. Tapia, V. Rivas, L. Nogués, E. Lucas, R. Vila-Bedmar, C. Murga, F. Mayor, Jr., Roles of GRK2 in cell signaling beyond GPCR desensitization: GRK2-HDAC6 interaction modulates cell spreading and motility. Sci. Signal. 5, pt3 (2012). 